tosa por instilación endovesical de BCG. Actas Uro Esp 2006; 30: 839842.

4. Lamm DL. Optimal BCG treatment of superficial bladder cancer as defined by American trials. Eur Uro 1992; 2: 12-6.

5. Martin Escudero JC, Pérez Paredes G, Ascensio Sánchez T, Herreros Fernández V. Neumonitis granulomatosa por BCG. An Med Interna (Madrid) 2003; 20: 57

6. Gupta RC, Lavengood R, Smith JP. Miliary tuberculosis due to intravesical Bacillus Calmette Guérin therapy. Chest 1998; 94: 1296-98.

7. Díaz C, Baldo C, Martín A, Fernández MJ, Muñoz M, Rodríguez L, et al. Tuberculosis parotidea secundaria a instilación vesical con BCG. Acta Otorrinolaringol Esp 2003; 54: 129-133.

8. Lamm DL, Vand der Meijden APM, Morales A, Brosman SA, Catalona WJ, Herr Hw, et al. Incidence and treatment of complications of bacillus Calmette-Guérin intravesical therapy in superficial bladder cancer. J Urol 1992; 147: 695-600.

9. Gilbert David N, Moellering Robert C, Sande Merle A. $19^{\text {a }}$ edition the Stanford Guide to antimicrobial therapy; 2006.

\section{Insuficiencia cardiaca de causa infecciosa}

\section{Sr. Director:}

La pericarditis purulenta es una rara localización infecciosa, pudiéndose producir por diseminación local o vía hematógenal. Siendo el absceso pericárdico una complicación excepcional de una pericarditis purulenta.

Presentamos un caso de un varón de 81 años de edad con intervención quirúrgica dos años antes de una valvulopatía aórtica con colocación de válvula protésica mecánica, posteriormente en clase funcional I de la New York Heart Association (NYHA), con ecocardiograma con válvula protésica normofuncionante sin otras alteraciones.

El paciente ingresa con una colecistitis gangrenada con plastrón perivesicular siendo intervenido de urgencia realizando una colecistectomía y limpieza quirúrgica del lecho vesicular, con cultivo del absceso peri-vesicular positivo para E. coli. El paciente presenta de forma progresiva mejoría del cuadro infeccioso y es dado de alta.

Reingresa varios días mas tarde con disnea y fiebre de $38,5^{\circ} \mathrm{C}$. En la exploración física se constata ingurgitación yugular, ruidos protésicos normofuncionantes y edema generalizado en situación de anasarca. Se inicia tratamiento depletivo con furosemida y antibiótico con imipenem. Se realiza hemocultivos seriados y una tomografía computerizada tóraco-abdominal (Fig. 1) constatando colección en lecho vesicular y subhepática de 4 x 6 x $5 \mathrm{~cm}$. Además se observó una colección paracardiaca pegada a ventrículo izquierdo de $5 \times 7 \mathrm{~cm}$ que comprimía el ventrículo izquierdo. En la ecocardiografía transtorácica se detecta una colección paracardiaca, con prótesis aortica normofuncionante sin evidencia de verrugas endomiocárdicas. Se realizó toracotomía de urgencia con drenaje de la colección intrapericárdica de aspecto serohemorrágico con cultivo positivo para E. coli, colocándose drenaje externo, además se realizó drenaje percutáneo de la colección intraabdominal. El paciente evolucionó satisfactoriamente desapareciendo la fiebre y resolviendose la situación de anasarca. Los hemocultivos fueron positivos para Bacteroides fragilis. Con el

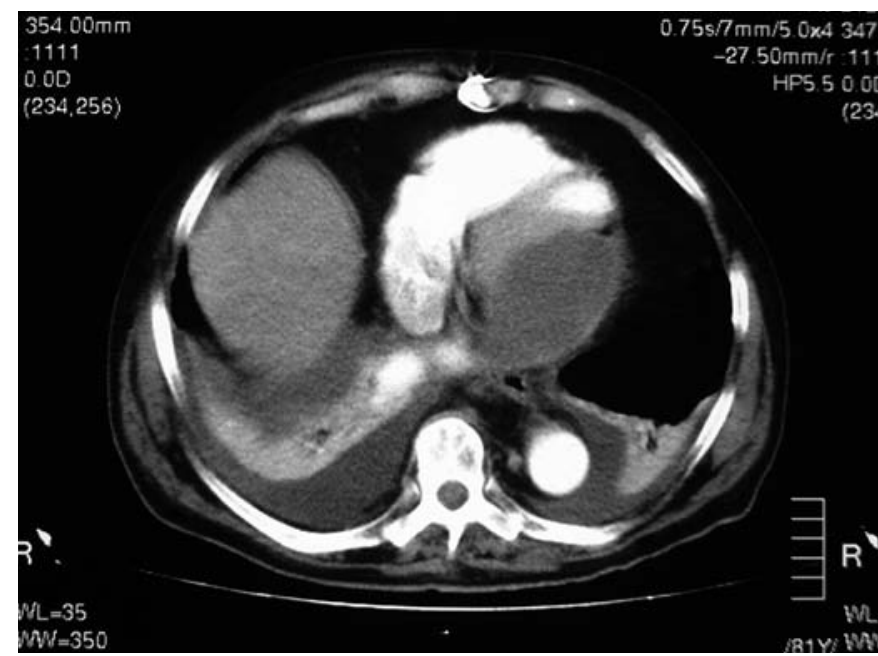

Fig. 1.

diagnóstico final de absceso pericardico por $E$. coli secundario a absceso intraabdominal.

En nuestro caso la infección por E. coli se produjo por diseminación local a través del diafragma cultivándose secuencialmente en absceso abdominal y pericárdico. Es plausible, que como ocurria en nuestro paciente, la cirugía cardiaca pueda dar lugar a formación de adherencias pericárdicas que favorezcan esta manifestación de debut (2). Las dos manifestaciones principales de los abscesos pericárdicos son la insuficiencia cardiaca restrictiva (especialmente en abscesos localizados adyacentes a aurículas o ventrículo derecho, y más difícil cuando lo hacen sobre ventrículo izquierdo) y el cuadro séptico asociado $(3,4)$. Nuestro paciente presentaba un absceso pegado a la pared libre del ventrículo izquierdo que comprimía hasta casi colapsar al ventrículo en diástole originándole insuficiencia cardiaca grave con situación de anasarca y cuadro séptico asociado. El tratamiento pasa siempre por un abordaje diagnóstico y terapéutico con punción o drenaje quirúrgico abierto y tratamiento antibiótico según el gérmen aislado. En nuestro caso además se realizó un drenaje percutáneo de la colección intraabdominal con resolución del cuadro infeccioso y resolución de la insuficiencia cardiaca.

\section{Fuentes Pardo, M. Belhassen García, J. Pardo Lledías, A. Carpio Pérez, I. Cruz ${ }^{1}$}

Servicios de Medicina Interna III y de ${ }^{I}$ Cardiología. Hospital Universitario. Salamanca

1. Nwiloh JO, Egbe PA, Tagoe AT, Weaver LW. Staphylococcus aureus Pericarditis Masquerading as Anterior Mediastinal:mediastinal mass from pericarditis. Chest 2000; 118; 1832-1833.

2. Mitsuma W, Ito M, Fujita S, Tsuchida K, Takahashi M, Hayashi J, Kodama M, Aizawa Y. Pericardial abscess detected by gallium- 67 scintigraphy 40 years after cardiac surgery. Int Heart J 2005; 46: 1119-22.

3. Caliskan K, Galema TW, Klootwijk AP. Tuberculous pericardial abscess causing right ventricular obstruction. Heart 2004; 90: 1041.

4. Hegde AN, Desai SB, Shivdasani B. Tuberculous pericardial abscess Eur J Cardiothorac Surg 2005; 28: 166. 